\title{
Constitutive phosphorylation of the FOXO1A transcription factor as a prognostic variable in gastric cancer
}

\author{
Ji Hun Kim${ }^{1}$, Min Kyu Kim², Hee Eun Lee ${ }^{1}$, Sung Jin $\mathrm{Cho}^{2}$, Yu Jin $\mathrm{Cho}^{2}$, Byung Lan Lee ${ }^{2,3}$, \\ Hye Seung Lee ${ }^{4}$, Seon Young Nam ${ }^{5}$, Jae-Seon $\mathrm{Lee}^{6}$ and Woo Ho Kim ${ }^{1,3}$ \\ ${ }^{1}$ Department of Pathology, Seoul National University College of Medicine, Seoul, Korea; ${ }^{2}$ Department of \\ Anatomy, Seoul National University College of Medicine, Seoul, Korea; ${ }^{3}$ Cancer Research Institute, Seoul \\ National University College of Medicine, Seoul, Korea; ${ }^{4}$ Department of Pathology, Seoul National University \\ Bundang Hospital, Gyeonggi, Korea; ${ }^{5}$ Division of Radiation Effect Research, Radiation Health Research \\ Institute, Korea Hydro \& Nuclear Power Co., Seoul, Korea and ${ }^{6}$ Radiological \& Medical Research Center, \\ Korea Institute of Radiological \& Medical Sciences, Seoul, Korea
}

\begin{abstract}
Increased phosphorylation of FOXO1A, a FOXO transcription factor, has been implicated in several human cancers; however, it has not been studied in the gastric cancer to date. To determine the status of pFOXO1A expression in human gastric cancers and to determine its relationship with other tumor-associated proteins, we performed immunohistochemical staining on tissue array slides containing 272 human gastric carcinoma specimens. In non-neoplastic gastric mucosa, the expression of pFOXO1A was observed primarily in cells in the proliferative zone and in areas of intestinal metaplasia. In gastric carcinomas, the expression of pFOXO1A was observed in 230 (84.6\%) out of 272 cases examined, and was positively correlated with the Ki-67-labeling index $(P=0.026)$. The expression of pFOX01A was higher in the early stages of pTNM $(P<0.001)$, and was inversely correlated with the intestinal type by Lauren's classification $(P=0.001)$, lymphatic invasion $(P=0.017)$ and lymph node metastasis $(P<0.001)$. Moreover, the expression of pFOXO1A was correlated with a longer patient survival $(P=0.004)$. In addition, the expression of pFOX01A was correlated with that of pAKT1 $(P<0.001)$, PTEN $(P=0.009)$, CDKN2A $(P=0.012)$, APC $(P=0.048)$, SMAD4 $(P<0.001)$, CD82 $(P=0.011)$, and BCL2 $(P=0.011)$. In conclusion, our results showed that the expression of pFOXO1A is a frequent and early event in gastric tumorigenesis and that there is a significant correlation between pFOXO1A and better prognosis. Thus, our data suggest that the expression of pFOX01A may serve as a valuable prognostic variable in gastric carcinoma and have significant implications for the development and application of targeted therapy. Modern Pathology (2007) 20, 835-842; doi:10.1038/modpathol.3800789; published online 11 May 2007
\end{abstract}

Keywords: FOXO1 protein; stomach cancer; survival analysis; tissue array analysis; immunohistochemistry

The forkhead transcription factors are DNA-binding proteins characterized by the presence of a conserved 110-amino-acid winged helix DNA-binding domain. ${ }^{1}$ The FOXO (Forkhead box, class O) is a subfamily of the forkhead transcription factor and consists of FOXO1A (FKHR: Forkhead in rhabdomyosarcoma, also known as FOXO1), FOXO3A

Correspondence: Dr WH Kim, MD, PhD, Department of Pathology, Seoul National University College of Medicine, 28 Yongon-dong, Chongno-gu, Seoul 110-799, Korea.

E-mail: woohokim@snu.ac.kr or

Dr BL Lee, MD, PhD, Department of Anatomy, Seoul National University College of Medicine, 28 Yongon-dong, Chongno-gu, Seoul 110-799, Korea.

E-mail: dslanat@snu.ac.kr

Received 11 January 2007; revised 11 March 2007; accepted 15 March 2007; published online 11 May 2007
(FKHR-like 1), and MLLT7 (AFX: acute-lymphocytic-leukaemia-1 fused gene from chromosome X, also known as FOXO4). Since each FOXO gene has a unique pattern of expression in tissues ${ }^{2,3}$ and exhibits a distinct response under a variety of conditions, ${ }^{4-6}$ their roles might be cell-type-specific.

The function of FOXO1A in mammalian cancer cells has been most frequently evaluated in in vitro studies which demonstrated that phosphorylated FOXO1A (pFOXO1A) is associated with increased cell survival in prostate cancer cells, ${ }^{7}$ glioma cells ${ }^{8}$ and multiple myeloma cells ${ }^{9}$ as well as being associated with the proliferation of multiple myeloma cells ${ }^{9}$ and colon cancer cells. ${ }^{10}$ In the in vivo studies using human cancer tissues, the expression of pFOXO1A has been correlated with an unfavorable outcome in acute myeloid leukemia ${ }^{11}$ as well as 
tumor progression and metastasis in colon cancer. ${ }^{12}$ In contrast, no significant correlation has been noted between pFOXO1A and any of the tumor characteristics of breast cancer. ${ }^{13}$

AKT1 (Akt, also known as protein kinase B) is a membranous protein kinase and is activated by phosphatidylinositol-triphosphate ${ }^{3-5}$ that is generated by active phosphatidylinositol-3 kinase (PI3K). ${ }^{14}$ PTEN, a lipid phosphatase, inactivates AKT1 by removing the D3 phosphate from the inositol ring of phosphatidylinositol ${ }^{3-5}$-triphosphate. ${ }^{15}$ It has been shown that FOXO transcription factors are regulated by AKT1 through direct phosphorylation of two or three conserved residues (Thr24, Ser256, and Ser319 in FOXO1A); ${ }^{16}$ this is followed by their interaction with YWHAQ (14-3-3) proteins, sequestration in the cytoplasm and suppression of the transcription of their target genes. ${ }^{17}$ However, the relationship between AKT1 and FOXO1A in human cancer is not well known.

Gastric cancer is one of the most common malignancies worldwide and the major cause of cancer related death in Asian countries. However, the status of FOXO1A phosphorylation and its biological significance in human gastric cancer have not been investigated to date. In the present study, we evaluated the expression of pFOXO1A in 272 surgically excised human gastric carcinoma tissues using immunohistochemistry performed on tissue array slides. In addition, we analyzed the association between the expression of pFOXO1A and prognosis as well as a number of clinicopathological factors. We also analyzed the correlation between the expression of pFOXO1A and that of antioxidant enzymes, pAKT1, tumor suppressor genes, or BCL2 as well as proliferation or apoptosis.

\section{Materials and methods}

\section{Patients and Samples}

The files of 272 surgically resected gastric cancer cases examined at the Department of Pathology, Seoul National University College of Medicine from 1 January to 30 June 1995 were analyzed. Age, sex, tumor location, and pTNM stage were evaluated by reviewing the medical records and pathological reports. ${ }^{18}$ The mean age of the patients was 54.8 years, and $93.3 \%$ of the patients had undergone curative resection. The cases enrolled in this study included 193 advanced and 79 early gastric carcinomas. According to the UICC criteria, there were 112 cases in stage I, 53 cases in stage II, 63 cases in stage III, and 44 cases in stage IV. No patient had received preoperative chemotherapy or radiotherapy. Glass slides were reviewed to determine histological type according to the WHO and Lauren's classification. This series included 102 intestinal types, 166 diffuse types, and four mixed types. Clinical outcomes were followed from the date of surgery to either the date of death or 1 December
2000, resulting in the follow-up period ranged from 1 to 72 months (mean, 51 months). Cases lost to follow-up and those resulting in death from any cause other than gastric cancer were censored for survival rate analysis. This protocol was reviewed and approved by the Institutional Review Board of Seoul National University (Approval No. C-0511519-163).

\section{Tissue Array Methods}

Six array blocks containing a total of 272 tissue cores obtained from patients with a gastric cancer were prepared as described previously (Superbiochips Laboratories, Seoul, Korea). ${ }^{19}$ Briefly, core tissue biopsies (2 $\mathrm{mm}$ in diameter) were taken from individual paraffin-embedded gastric tumors (donor blocks) and arranged in the new recipient paraffin blocks (tissue array block) using a trephine apparatus. As we have reported previously, ${ }^{20}$ the staining results of the different intratumoral areas of gastric carcinomas in these tissue array blocks showed an excellent agreement. A core was chosen from each case for analysis. We defined an adequate case as a tumor occupying more than $10 \%$ of the core area. Each block contained internal controls consisting of non-neoplastic gastric mucosa from body, antrum, and other areas showing intestinal metaplasia. Sections of $4 \mu \mathrm{m}$ thickness were cut from each tissue array block, deparaffinized, and dehydrated.

\section{Immunohistochemistry}

Immunohistochemical staining for pFOXO1A was performed as described previously ${ }^{18}$ using a streptavidin peroxidase procedure (avidin-biotin complex method) after antigen retrieval using an autoclave. Anti-phospho-FOXO1A (Ser256) from Cell Signaling Technology (Beverly, MA, USA) was used as the primary antibody. Other antibodies were purchased from the following companies: anti-manganese superoxide dismutase (MnSOD) (1:300) and anticopper/zinc superoxide dismutase (Cu/ZnSOD) (1:2000) from StressGen Biotechnologies Co. (Victoria, BC, Canada); anti-glutathione S-transferase-pi (GSTP1) (1:5000) from Novocastra Laboratories Ltd, New castle, United Kingdom; anti-AKT1 (1:50) and anti-phospho-AKT1 (Ser 473) (1:100) from New England Biolabs (Beverly, MA, USA); anti-CDKN2A (p16) (1:50) from PharMingen (San Diego, CA, USA); anti-adenomatous polyposis coli (APC) (1:400), antiSMAD4 (1:50), and anti-CD82 (KAI1) (1:200) from Santa Cruz Biotechnology (Santa Cruz, CA, USA); anti-Ki-67 (1:50) and anti-BCL2 (1:100) from DAKO Corporation (Carpinteria, CA, USA); and anti-PTEN (1:50) from A.G. Scientific, Inc. (San Diego, CA, USA). Other chemicals were purchased from Sigma (St Louis, MO, USA). The results of immunostaining were evaluated by two pathologists (JHK and HSL), who were blinded to the origin of the samples. The 
concordance rates were generally high ranging from $75.7 \% \quad(\kappa=0.328, P<0.001)$ to $87.5 \% \quad(\kappa=0.591$, $P<0.001)$. Discrepancies were resolved by reaching consensus. For statistical analysis, the results of immunostaining for proteins other than Ki-67 were considered as positive if $10 \%$ or more of the neoplastic cells were stained. For Ki-67 staining, we evaluated 300 cells and counted the cells with nuclear staining for each specimen. The proliferation index was defined as follows: proliferation index $(\%)=100 \times \mathrm{Ki}-67$-positive cells/total cells.

\section{TUNEL Staining}

Apoptotic cells were identified by the terminal deoxynucleotidyl transferase mediated deoxyuridine triphosphate biotin nick end labeling (TUNEL) method using an apoptosis detection kit (Chemicon International, Temecula, CA, USA). Labeling procedures were performed according to the manufacturer's instruction, which was followed by staining with diaminobenzine for $2 \mathrm{~min}$ and counterstaining with hematoxylin. In total, 300 cells were counted for each specimen. The apoptosis index was defined as follows: apoptosis index $(\%)=100 \times$ apoptotic cells/total cells.

\section{Statistical Analysis}

All statistical analyses were conducted using SPSS version 11.0 software (SPSS, Chicago, IL, USA).

The relationships between pFOXO1A expression and the apoptosis or cell proliferation index were analyzed using the two-tailed Student's $t$-test. To determine the significance of correlation between

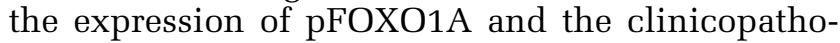
logical factors or the expression of other proteins, either the $\chi^{2}$ test or the Fisher's exact test (two-sided) was performed. Survival curves were estimated using the Kaplan-Meier product-limit method, and the significance of differences between the survival curves was determined using the log-rank test. To determine whether the expression of pFOXO1A is an independent prognostic variable, multivariate survival analysis was performed using the Cox proportional hazard model. The results were considered statistically significant when $P$ values were less than 0.05 .

\section{Results}

\section{Expression of pFOXO1A in Gastric Cancers}

In non-neoplastic gastric mucosa, pFOXO1A was expressed throughout the proliferative zone of the gastric glands (Figure 1a) and in the areas showing intestinal metaplasia (Figure 1b). The expression of pFOXO1A was observed in the cytoplasm and at times in the nucleus. Tumors expressing pFOXO1A also showed positive staining in the cytoplasm and intermittently in the nucleus, whereas the surrounding stromal cells exhibited little or no staining (Figure 1c). Cells showing cytoplasmic staining, regardless of the presence of nuclear staining, were considered to exhibit constitutive FOXO1A phosphorylation. The expression of pFOXO1A was found in $84.6 \%$ of gastric tumors.

\section{Association between the Expression of pFOXO1A and Clinicopathological Factors}

Data representing the correlation between the expression of pFOXO1A and the clinicopathological features of the 272 gastric cancer cases are summarized in Table 1. The expression of pFOXO1A was more likely to be found in the intestinal type by Lauren's classification $(P=0.001)$ and in the early pTNM stages $(P<0.001)$. Sixty-five percent of pFOXO1A positive tumors were in pTNM stages I and II, while $35 \%$ were in stages III and IV. Moreover, we found an inverse correlation between

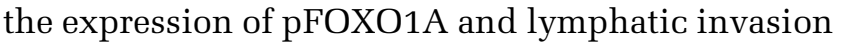
$(P=0.017)$ or lymph node metastasis $(P<0.001)$. No association was found between the expression of $\mathrm{pFOXO1A}$ and age, gender, tumor location, or distant metastasis.

\section{Association between the Expression of pFOXO1A and Proliferation or Apoptosis}

The results of the immunohistochemistry for Ki-67 and TUNEL staining are shown in Table 2. The percentages of Ki-67-positive cells and apoptotic cells were variable. The expression of pFOXO1A significantly correlated with the proliferation index (evaluated by Ki-67 labeling) but not with apoptosis index (evaluated by TUNEL staining).

\section{The Expression of pFOXO1A in Relation to the Expression of Antioxidant Enzymes}

We examined whether the expression of pFOXO1A correlates with that of antioxidant enzymes in gastric carcinoma tissues. The expression of pFOXO1A did not correlate with that of antioxidant enzymes such as MnSOD $(P=0.210), \mathrm{Cu} / \mathrm{ZnSOD}$ $(P=0.411)$, or GSTP1 $(P=0.438)$.

\section{The Expression of pFOXO1A in Relation to the Expression of pAKT1}

We examined whether the expression of pFOXO1A correlates with that of pAKT1 in gastric carcinoma tissues (Table 3). The expression of pFOXO1A was found to correlate with that of pAKT1 $(P<0.001)$. On the contrary, no association was found between the expression of $\mathrm{pFOXO1A}$ and that of AKT1 $(P=0.128)$. 

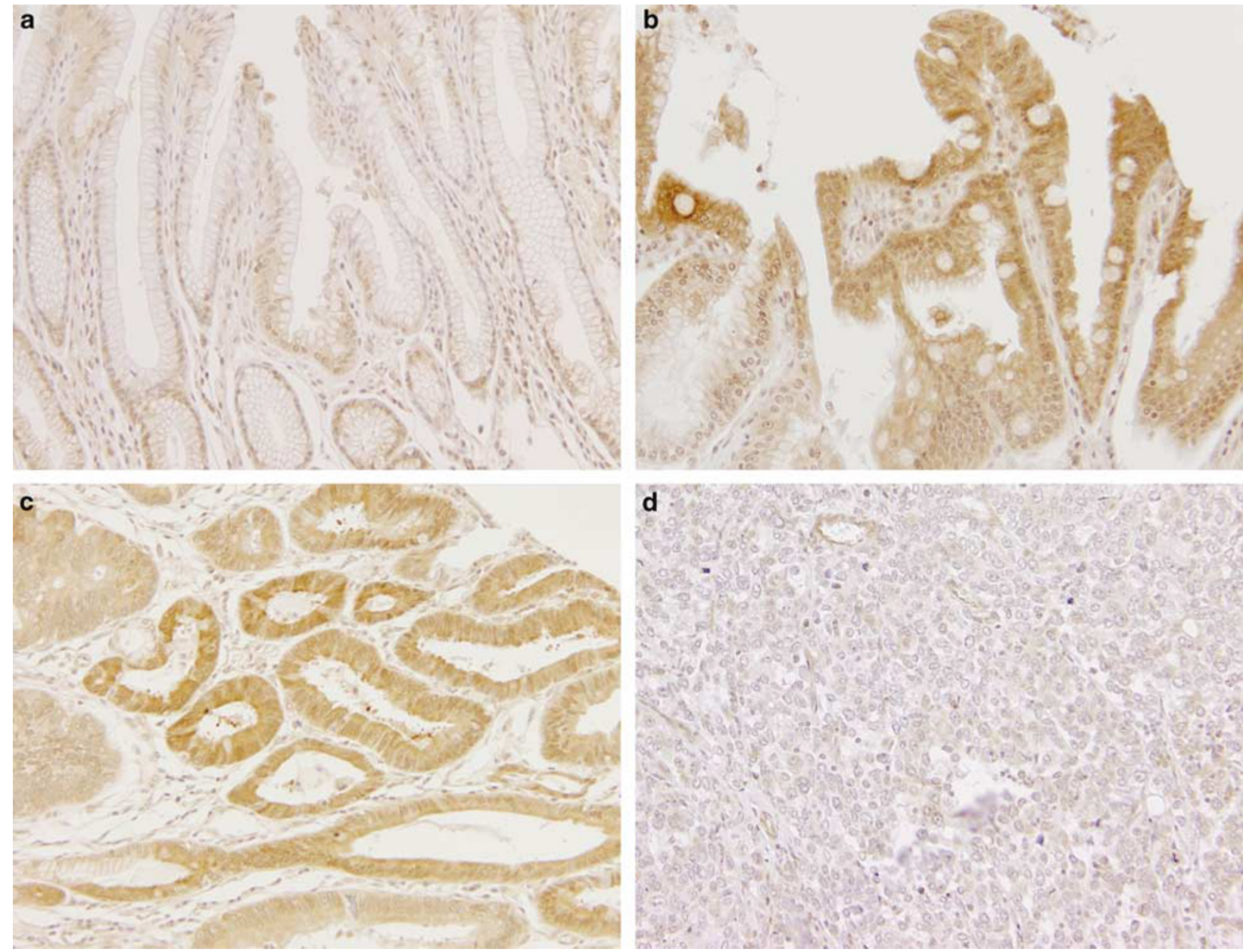

Figure 1 Representative findings of the immunohistochemistry for pFOXO1A. pFOXO1A was expressed in the proliferative zone in normal gastric mucosa $(\mathbf{a}, \times 100)$ and in areas of intestinal metaplasia $(\mathbf{b}, \times 200)$. Gastric carcinoma cells showing cytoplasmic pFOXO1A expression with or without nuclear staining $(\mathbf{c}, \times 200)$. Gastric carcinoma cells without pFOXO1A expression $(\mathbf{d}, \times 200)$.

\section{Correlation between the Expression of pFOXO1A and Expression of Other Tumor-Associated Proteins}

The correlation between the expression of pFOXO1A or pAKT1 and other tumor-associated proteins are summarized in Table 4. The expression of pFOXO1A correlated with that of PTEN $(P=0.009)$, CDKN2A $(P=0.012)$, APC $(P=0.048)$, SMAD4 $(P<0.001)$, CD82 $(P=0.011)$, and BCL2 $(P=0.011)$. Similarly, the expression of pAKT1 also correlated with that of tumor suppressor genes such as CDKN2A $(P=0.004)$, APC $(P=0.002)$, SMAD4 $(P<0.001)$, and CD82 $(P<0.001)$. However, no association was found between the expression of pAKT1 and that of PTEN or BCL2.

\section{The Expression of pFOXO1A in Relation to Prognosis}

Of the 272 patients analyzed, those with the expression of pFOXO1A (224 cases) showed a significantly higher survival rate than those without its expression ( $P=0.004$; Figure 2a). In the analysis of the combined status of the expression of
pFOXO1A and lymph node metastasis in relation to prognosis, patients with a pFOXO1A-positive and lymph node-negative tumor showed significantly better outcome than those with the other combinations $(P<0.0001$; Figure 2b). Moreover, among the patients with lymph node-negative tumors, the pFOXO1A-positive subgroup exhibited a better survival than the pFOXO1A-negative subgroup $(P=0.036)$. However, multivariate Cox regression analysis including the pTNM stage revealed that the expression of pFOXO1A was not an independent prognostic factor.

\section{Discussion}

The present study analyzed the expression of pFOXO1A and its biological significance in human gastric carcinoma for the first time. We found that pFOXO1A was expressed in $84.6 \%$ of gastric cancer specimens, and the expression of pFOXO1A was associated with a good prognosis as well as specific clinicopathological factors. 
Table 1 Correlation between the expression of pFOXO1A and clinicopathological factors in human gastric cancer

\begin{tabular}{|c|c|c|c|}
\hline & $\begin{array}{c}\text { pFOXO1A } \\
\text { positive (\%) }\end{array}$ & $\begin{array}{c}\text { pFOXO1A } \\
\text { negative (\%) }\end{array}$ & $\mathrm{P}$-value \\
\hline Total $(n=272)$ & $230(85)$ & $42(15)$ & \\
\hline \multicolumn{4}{|l|}{ Age (years) } \\
\hline $0-39$ & $37(90)$ & $4(10)$ & 0.495 \\
\hline $40-65$ & $143(83)$ & 29 (17) & \\
\hline $66-99$ & $50(85)$ & $9(15)$ & \\
\hline \multicolumn{4}{|l|}{ Gender } \\
\hline Male & $158(86)$ & $26(14)$ & 0.387 \\
\hline Female & $72(82)$ & $16(18)$ & \\
\hline \multicolumn{4}{|l|}{ Location } \\
\hline Antrum & $121(85)$ & $22(15)$ & 0.978 \\
\hline Body and cardia & $109(84)$ & $20(16)$ & \\
\hline \multicolumn{4}{|c|}{ Lauren's classification } \\
\hline Intestinal & $96(94)$ & $6(6)$ & 0.001 * \\
\hline Diffuse & $131(79)$ & $35(21)$ & \\
\hline Mixed & $3(75)$ & $1(25)$ & \\
\hline \multicolumn{4}{|l|}{ TNM stage } \\
\hline I & $107(96)$ & $5(4)$ & $<0.001^{*}$ \\
\hline II & 42 (79) & $11(21)$ & \\
\hline III & $49(78)$ & $14(22)$ & \\
\hline IV & $32(73)$ & $12(27)$ & \\
\hline \multicolumn{4}{|l|}{ Lymphatic invasion } \\
\hline Absent & $168(88)$ & $23(12)$ & $0.017^{*}$ \\
\hline Present & $62(77)$ & $19(23)$ & \\
\hline \multicolumn{4}{|c|}{ Lymph node metastasis } \\
\hline Absent & $94(96)$ & $4(4)$ & $<0.001^{*}$ \\
\hline Present & $136(78)$ & $38(22)$ & \\
\hline \multicolumn{4}{|l|}{ Distant metastasis } \\
\hline Absent & $220(86)$ & 37 (14) & 0.063 \\
\hline Present & $10(67)$ & $5(33)$ & \\
\hline
\end{tabular}

*Considered to be statistically significant.

Table 2 Correlation between the expression of pFOXO1A and the proliferation index and the apoptosis index in human gastric cancer

\begin{tabular}{lccc}
\hline & \multicolumn{2}{c}{ pFOXO1A } & P-value \\
\cline { 2 - 3 } & $\begin{array}{c}\text { Positive (n=230) } \\
\text { Mean } \pm \text { s.d. (\%) }\end{array}$ & $\begin{array}{c}\text { Negative (n=42) } \\
\text { Mean } \pm \text { s.d. (\%) }\end{array}$ & \\
\hline Ki-67 (\%) & $23.81 \pm 20.39$ & $15.36 \pm 20.28$ & $0.025^{*}$ \\
TUNEL (\%) & $3.26 \pm 2.47$ & $3.28 \pm 2.58$ & 0.958 \\
\hline
\end{tabular}

${ }^{*}$ Considered to be statistically significant.

In the present study, pFOXO1A was expressed throughout the proliferative zone and in areas showing intestinal metaplasia in the non-neoplastic gastric mucosa. Because the intestinal metaplasia shows a higher proliferation index than the normal gastric mucosa, ${ }^{21}$ this expression pattern suggests a positive association between the expression of
Table 3 The expression of pFOXO1A in relation to the expression of pAKT1 and AKT1

\begin{tabular}{lccc}
\hline & $\begin{array}{c}\text { pFOXO1A } \\
\text { positive (\%) }\end{array}$ & $\begin{array}{c}\text { pFOXO1A } \\
\text { negative (\%) }\end{array}$ & P-value \\
\hline pAKT1 & & & \\
$\quad$ Positive & $183(90)$ & $21(10)$ & $<0.001^{*}$ \\
Negative & $39(65)$ & $21(35)$ & \\
AKT1 & & & \\
$\quad$ Positive & $31(91)$ & $4(9)$ & 0.128 \\
Negative & $191(84)$ & $37(16)$ & \\
\hline
\end{tabular}

${ }^{*}$ Considered to be statistically significant.

pFOXO1A and cell proliferation. Moreover, pFOXO1A expression was positively correlated with the Ki-67-labeling index $(P=0.026)$. Thus, our data are consistent with the results of previous studies in which the expression of pFOXO1A was associated with cell proliferation of the multiple myeloma cells $^{9}$ and colon cancer cells. ${ }^{10}$ On the other hand, we found that pFOXO1A expression was not correlated with either the apoptosis index $(P=0.737)$ or expression of the antioxidant enzymes such as MnSOD, Cu/ZnSOD, and GSTP1 $(P=0.210$, 0.411 , and 0.438 , respectively). Taken together, the data presented here show that, among the reported functions of FOXO proteins such as induction of apoptosis, ${ }^{7}$ cell cycle arrest, ${ }^{22}$ and protection from oxidative stress, ${ }^{23} \mathrm{FOXO} 1 \mathrm{~A}$ activity in gastric cancer may be related to cell cycle arrest.

The results of the present study suggest that the expression of pFOXO1A is an early event of gastric tumorigenesis because it is associated with early pTNM stages and absence of lymphatic invasion or lymph node metastasis. Our results disagree with other studies, which reported that the expression of pFOXO1A is associated with an unfavorable outcome in acute myeloid leukemia ${ }^{11}$ or with the advanced stage of colon cancer. ${ }^{12}$ Thus, the biological significance of the expression of pFOXO1A in the cancer appears to vary depending on the cancer cell type.

Lymph node metastasis is one of the most important factors in determining the prognosis of gastric carcinomas. $^{24}$ In the present study, the pFOXO1A-positive and lymph node metastasisnegative combination showed a significant survival advantage over the other combinations. Among the patients without lymph node metastasis, patients with a pFOXO1A-positive tumor exhibited a better survival than those with a pFOXO1A-negative tumor $(P=0.0036)$. Thus, our results strongly suggest that the expression of pFOXO1A is an important prognostic factor regardless of lymph node metastasis, at least in the early stages of gastric cancers.

Although FOXO1A is generally regarded as a direct substrate of $\mathrm{AKT} 1,{ }^{16}$ it remains controversial 
Table 4 The expression of pFOXO1A and pAKT1 in relation to the expression of other tumor-associated proteins

\begin{tabular}{|c|c|c|c|c|c|c|}
\hline & \multicolumn{2}{|c|}{ pFOXO1A } & \multirow[t]{2}{*}{ P-value } & \multicolumn{2}{|c|}{$p A K T 1$} & \multirow[t]{2}{*}{ P-value } \\
\hline & Positive (\%) & Negative (\%) & & Positive (\%) & Negative (\%) & \\
\hline \multicolumn{7}{|l|}{ PTEN } \\
\hline Positive & $181(87)$ & $26(13)$ & $0.009^{*}$ & $187(80)$ & $47(20)$ & 0.076 \\
\hline Negative & 41 (73) & $15(27)$ & & 43 (69) & $19(31)$ & \\
\hline \multicolumn{7}{|l|}{$C D K N 2 A$} \\
\hline Positive & 160 (89) & 20 (11) & $0.012^{*}$ & 168 (83) & 35 (17) & $0.004^{*}$ \\
\hline Negative & $64(77)$ & $19(23)$ & & 64 (68) & $30(32)$ & \\
\hline \multicolumn{7}{|l|}{$A P C$} \\
\hline Positive & 176 (87) & 26 (13) & $0.048^{*}$ & $182(82)$ & 39 (18) & $0.002 *$ \\
\hline Negative & 46 (77) & $14(23)$ & & 47 (65) & $25(35)$ & \\
\hline \multicolumn{7}{|l|}{ SMAD4 } \\
\hline Positive & 201 (88) & $28(12)$ & $<0.001^{*}$ & 212 (81) & 50 (19) & $<0.001^{*}$ \\
\hline Negative & 21 (60) & $14(40)$ & & 21 (53) & $19(48)$ & \\
\hline \multicolumn{7}{|l|}{ CD82 } \\
\hline Positive & $199(94)$ & $12(6)$ & $0.011^{*}$ & 202 (86) & 32 (14) & $<0.001^{*}$ \\
\hline Negative & $29(50)$ & $29(50)$ & & $34(51)$ & $33(49)$ & \\
\hline \multicolumn{7}{|l|}{$B C L 2$} \\
\hline Positive & $29(100)$ & $0(0)$ & $0.011^{*}$ & 32 (86) & 5 (14) & 0.199 \\
\hline Negative & $195(83)$ & 40 (17) & & $203(77)$ & $60(23)$ & \\
\hline
\end{tabular}

${ }^{*}$ Considered to be statistically significant.
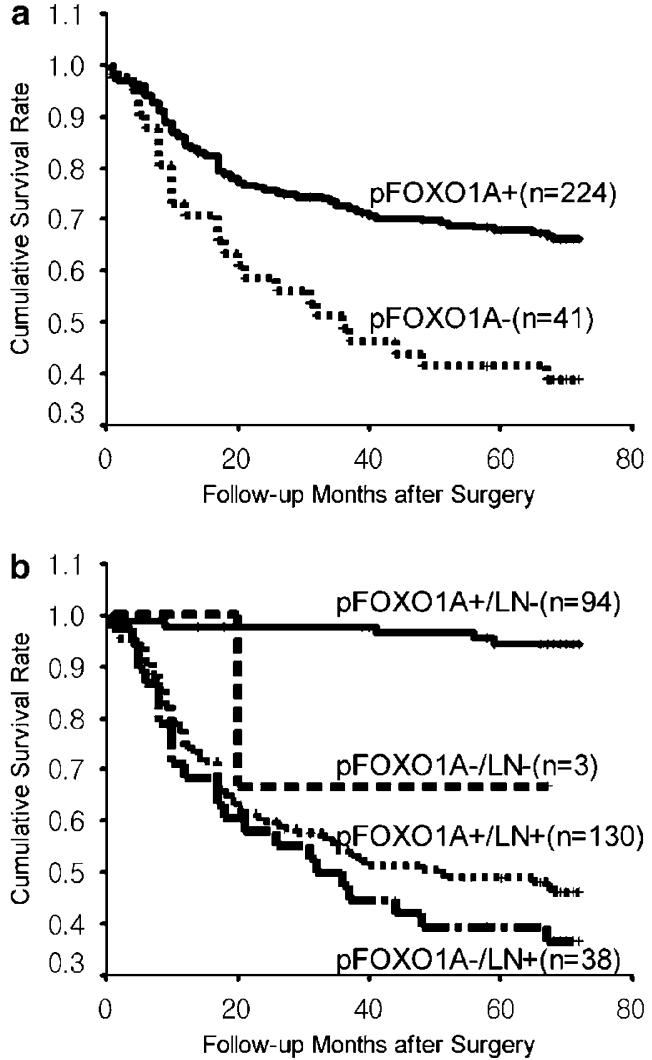

Figure 2 Kaplan-Meier curves for patient survival. (a) pFOXO1A-positive carcinomas showed a more favorable prognosis than pFOXO1A-negative carcinomas $(P=0.004)$. (b) Patients with pFOXO1A-positive and lymph node (LN)-negative combination showed the best outcome $(P<0.0001)$ whether FOXO1A is a downstream target of pAKT1 in human cancers. A positive association has been reported in glioblastoma, ${ }^{25}$ lung cancer ${ }^{26}$ and soft tissue sarcoma, ${ }^{27}$ while no association was observed in prostate cancer ${ }^{15}$ and in breast cancer. ${ }^{13}$ The present study showed a positive correlation between the expression of pFOXO1A and that of pAKT1 $(P<0.001)$. In addition, being consistent with our previous report as to $\mathrm{pAKT} 1,{ }^{19} \mathrm{pFOXO} 1 \mathrm{~A}$ correlates with early stages, absence of nodal metastasis and a better outcome. Thus, the phosphorylation of FOXO1A, which may be associated with AKT1 activation at least in part, appears to be more important for tumor initiation than tumor progression of gastric tumor.

We reported previously that the expression of pAKT1 correlates with that of tumor suppressor genes such as APC and SMAD4, but does not correlate with the expression of PTEN or BCL2. ${ }^{19}$ In the present study, we extended our examination of tumor suppressor genes and found positive associations of the expression of pAKT1 with that of CDKN2A $(P=0.004)$ and CD82 $(P<0.001)$. Meanwhile, the expression of pFOXO1A was shown to correlate with that of the above five tumor suppressor genes (APC, SMAD4, CDKN2A, CD82, and PTEN) and BCL2 (summarized in Table 4). Therefore, our data demonstrate a similarity between the expression of pFOXO1A and that of pAKT1 in terms of their correlation with the expression of tumor suppressor genes with the exception of their correlation with the expression of PTEN or BCL2. 
The explanation for this result is unclear. Possibly, the PTEN is involved in the phosphorylation of FOXO1A through a signaling pathway different from the AKT1-FOXO1A pathway, but we currently have no evidence to support this idea. In addition, the positive association between the expression of pFOXO1A and that of BCL2 is intriguing.

Although the present study showed positive associations between the expression of pFOXO1A and that of many other proteins with statistical significance, these positive associations should be interpreted with caution because of the potential artifact in the immunohistochemical study. The positive correlations in our archived tissue samples might have simply resulted from the presence of samples with suboptimal antigenicity, which show negative stain for one protein and negative stain for another protein. To clearly resolve this potential artifact, further molecular studies on the signaling pathways involving $\mathrm{AKT} 1$ and FOXO1A in gastric cancer are needed.

In conclusion, our data demonstrated that the expression of pFOXO1A is more frequent in the early stage gastric carcinomas and is significantly associated with the intestinal type by Lauren's classification, negative nodal status, and better prognosis. Moreover, the expression of pFOXO1A correlated significantly with that of pAKT1, BCL2 and other tumor suppressor genes including APC, SMAD4, CDKN2A, CD82, and PTEN. Although further investigations are needed to clarify the molecular mechanisms involved in FOXO1A phosphorylation, these findings suggest that the pFOXO1A, along with pAKT1, is a candidate molecular biomarker for the early gastric carcinoma. In addition, blocking the AKT1-FOXO1A pathway with appropriate inhibitors might be a promising approach for the development of targeted therapies.

\section{Acknowledgements}

This work was supported by FG-06-11-03 of the 21C Frontier Functional Human Genome Project from Ministry of Science and Technology of Korea. CSJ and CYJ were supported by the second stage Brain Korea 21 Project in 2006.

\section{References}

1 Kops GJ, Burgering BM. Forkhead transcription factors: new insights into protein kinase B (c-akt) signaling. J Mol Med 1999;77:656-665.

2 Furuyama T, Kitayama K, Shimoda Y, et al. Abnormal angiogenesis in FOXO1 (Fkhr)-deficient mice. J Biol Chem 2004;279:34741-34749.

3 Kitamura T, Nakae J, Kitamura Y, et al. The forkhead transcription factor Foxo1 links insulin signaling to Pdx1 regulation of pancreatic beta cell growth. J Clin Invest 2002;110:1839-1847.
4 Nakae J, Kitamura T, Kitamura Y, et al. The forkhead transcription factor Foxo1 regulates adipocyte differentiation. Dev Cell 2003;4:119-129.

5 Richards JS, Sharma SC, Falender AE, et al. Expression of FKHR, FKHRL1, and AFX genes in the rodent ovary: evidence for regulation by IGF-I, estrogen, and the gonadotropins. Mol Endocrinol 2002; 16:580-599.

6 Bois PR, Grosveld GC. FKHR (FOXO1A) is required for myotube fusion of primary mouse myoblasts. EMBO J 2003;22:1147-1157.

7 Modur V, Nagarajan R, Evers BM, et al. FOXO proteins regulate tumor necrosis factor-related apoptosis inducing ligand expression. Implications for PTEN mutation in prostate cancer. J Biol Chem 2002;277: 47928-47937.

8 Ciechomska I, Pyrzynska B, Kazmierczak P, et al. Inhibition of Akt kinase signalling and activation of Forkhead are indispensable for upregulation of FasL expression in apoptosis of glioma cells. Oncogene 2003;22:7617-7627.

9 Lentzsch S, Chatterjee M, Gries M, et al. PI3-K/AKT/ FKHR and MAPK signaling cascades are redundantly stimulated by a variety of cytokines and contribute independently to proliferation and survival of multiple myeloma cells. Leukemia 2004;18:1883-1890.

10 Kops GJ, Medema RH, Glassford J, et al. Control of cell cycle exit and entry by protein kinase B-regulated forkhead transcription factors. Mol Cell Biol 2002; 22:2025-2036.

11 Cheong JW, Eom JI, Maeng HY, et al. Constitutive phosphorylation of FKHR transcription factor as a prognostic variable in acute myeloid leukemia. Leuk Res 2003;27:1159-1162.

12 Bravou V, Klironomos G, Papadaki E, et al. ILK overexpression in human colon cancer progression correlates with activation of beta-catenin, down-regulation of E-cadherin and activation of the Akt-FKHR pathway. J Pathol 2006;208:91-99.

13 Bose S, Chandran S, Mirocha JM, et al. The Akt pathway in human breast cancer: a tissue-array-based analysis. Mod Pathol 2006;19:238-245.

14 Chan TO, Rittenhouse SE, Tsichlis PN. AKT/PKB and other D3 phosphoinositide-regulated kinases: kinase activation by phosphoinositide-dependent phosphorylation. Annu Rev Biochem 1999;68:965-1014.

15 Thomas GV, Horvath S, Smith BL, et al. Antibodybased profiling of the phosphoinositide 3-kinase pathway in clinical prostate cancer. Clin Cancer Res 2004; 10:8351-8356.

16 Fukunaga K, Ishigami T, Kawano T. Transcriptional regulation of neuronal genes and its effect on neural functions: expression and function of forkhead transcription factors in neurons. J Pharmacol Sci 2005; 98:205-211.

17 Burgering BM, Kops GJ. Cell cycle and death control: long live Forkheads. Trends Biochem Sci 2002;27: $352-360$.

18 Lee HS, Lee HK, Kim HS, et al. Tumor suppressor gene expression correlates with gastric cancer prognosis. J Pathol 2003;200:39-46.

19 Nam SY, Lee HS, Jung GA, et al. Akt/PKB activation in gastric carcinomas correlates with clinicopathologic variables and prognosis. APMIS 2003;111: 1105-1113.

20 Lee HS, Lee HK, Kim HS, et al. MUC1, MUC2, MUC5AC, and MUC6 expressions in gastric carcino- 
mas: their roles as prognostic indicators. Cancer 2001;92:1427-1434.

21 Lee KM, Lee DS, Yang JM, et al. Effect of Helicobacterpylori on gastric epithelial cell kinetics and expression of apoptosis-related proteins in gastric carcinogenesis. Korean J Gastroenterol 2003;42:12-19.

22 Schmidt M, Fernandez de Mattos S, van der Horst A, et al. Cell cycle inhibition by FOXO forkhead transcription factors involves downregulation of cyclin D. Mol Cell Biol 2002;22:7842-7852.

23 Essers MA, de Vries-Smits LM, Barker N, et al. Functional interaction between beta-catenin and FOXO in oxidative stress signaling. Science 2005;308: 1181-1184.
24 Adachi Y, Kamakura T, Mori M, et al. Prognostic significance of the number of positive lymph nodes in gastric carcinoma. Br J Surg 1994;81:414-416.

25 Choe G, Horvath S, Cloughesy TF, et al. Analysis of the phosphatidylinositol $3^{\prime}$-kinase signaling pathway in glioblastoma patients in vivo. Cancer Res 2003;63: 2742-2746.

26 Balsara BR, Pei J, Mitsuuchi Y, et al. Frequent activation of AKT in non-small cell lung carcinomas and preneoplastic bronchial lesions. Carcinogenesis 2004;25:2053-2059.

27 Tomita Y, Morooka T, Hoshida Y, et al. Prognostic significance of activated AKT expression in soft-tissue sarcoma. Clin Cancer Res 2006;12:3070-3077. 